\title{
Detection of important abnormalities of the differential count using the Coulter STKR blood counter
}

\author{
D F BARNARD, S A BARNARD, A B CARTER, S J MACHIN, K G PATTERSON, \\ A YARDUMIAN Department of Haematology, Middlesex Hospital, London
}

SUMMARY An assessment of the three part differential provided by the Coulter STKR blood counter showed good correlation when compared with an 800 cell manual differential. Satisfactory flagging of eosinophilia, basophilia, and the presence of immature cells was found. The use of variables derived from the STKR in conjunction with interpretive reporting and user-defined flagging enabled this department to reduce considerably the numbers of films requiring manual differential counts.

Modern automated counters allow blood counts to be processed rapidly with a minimum of time consuming manual examination of the blood film. The use of such counters introduces two potential problems. First, automated counters may miss abnormalities of clinical importance. Secondly, they measure variables not usually assessed by manual or semiautomated methods. Some of these are of uncertain clinical importance and may cause unnecessary anxiety to the clinician.

We assessed the efficiency of one such blood cell counter, the Coulter STKR, that provides a three part differential count and has the ability to indicate samples that have raised eosinophil or basophil counts or immature cells.

\section{Material and methods}

All samples were obtained by venepuncture and taken into Becton Dickinson Vacutainers containing $0.054 \mathrm{ml}$ of $0.34 \mathrm{M} \mathrm{K}_{3}$ edetic acid producing a final concentration of $1.5 \mathrm{mg} / \mathrm{ml}$. The samples were drawn from the routine laboratory workload with preferential selection of those samples known to or suspected of containing white cell abnormalities. The samples were analysed by the STKR and wedge blood films stained by May-Grünwald Giemsa within five hours of venepuncture.

A manual 800 cell differential was performed on all samples using the format of the NCCLS guidelines on assessing automated differential analysers. ${ }^{1}$ The guidelines were not followed in separately classifying stab or band cells, nor in the refereeing of disagreements between observers.

The Coulter S Plus IV and its derivative the Coulter STKR have been discussed elsewhere. ${ }^{2-4}$ Cells of

Accepted for publication 31 March 1989 volume greater than $35 \mathrm{fl}$ remaining after lysis of red cells are classified as leucocytes. The data terminal derives the three part differential from a histogram of white cell size plotted against relative numbers (fig 1). The three peaks on the histogram represent lymphocytes (35-90 fl), monocytes (90-160 fl), and granulocytes $(160-450 \mathrm{f})$. Failure of valley detection in certain areas of the white cell histogram will trigger region flags. These, in combination with other abnormalities detected by machine in the white cell histogram, generate "interpretive reports". These are narrative comments in plain language on the detected abnormalities.

The Auto-Reporter printer sorts result forms into two groups-normal and abnormal. Sorting criteria may be user-specified on the Auto-Reporter keypad.

A "positive" result on the STKR was defined as the presence of any of the "flags" as follows: (i) backlit variable; (ii) vote-out or out-of-range result; (iii) incomplete computation; (iv) region flag; (v) interpretive report.

No account was taken of any flagging that may be user-defined or of any flagging on the platelet or red cell variables, with the exception of "suspect NRBC" (nucleated red cells).

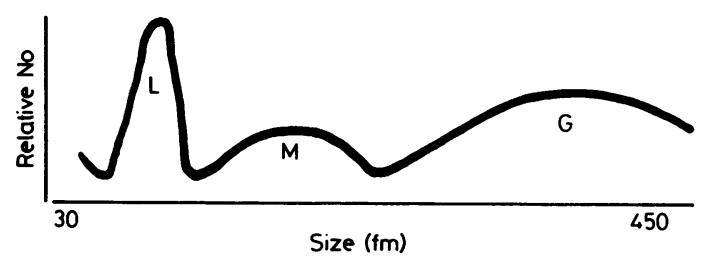

Fig 1 STKR leucocyte histogram. Leucocytes are classified on the basis of their size (horizontal axis) into granulocytes (large), monocytes (medium), and lymphocytes (small) size. 
The STKR does not provide a value for eosinophils or basophils but indicates that they are lower than $0.7 \times 10^{9} / 1$ for eosinophils or lower than $0.2 \times 10^{9} / 1$ for basophils. Values above these ranges are flagged by either or both the narrative statements:

\section{SUSPECT: EOS IMM GRANS ATYP LYMPHS \& BLASTS}

A manual result was "flagged" as "positive" if one or more immature cells were identified by any of the four observers on a 200 cell differential.

(a) Neutrophils

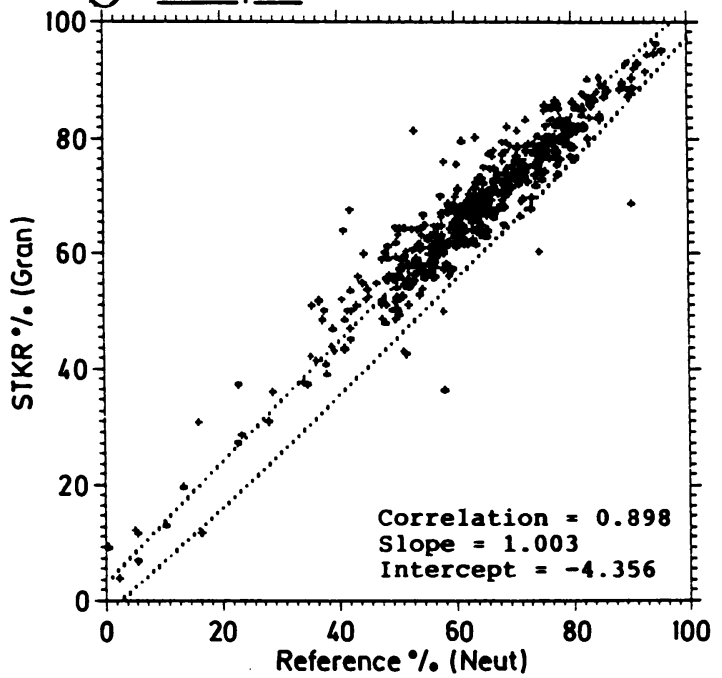

(C) Lymphocytes

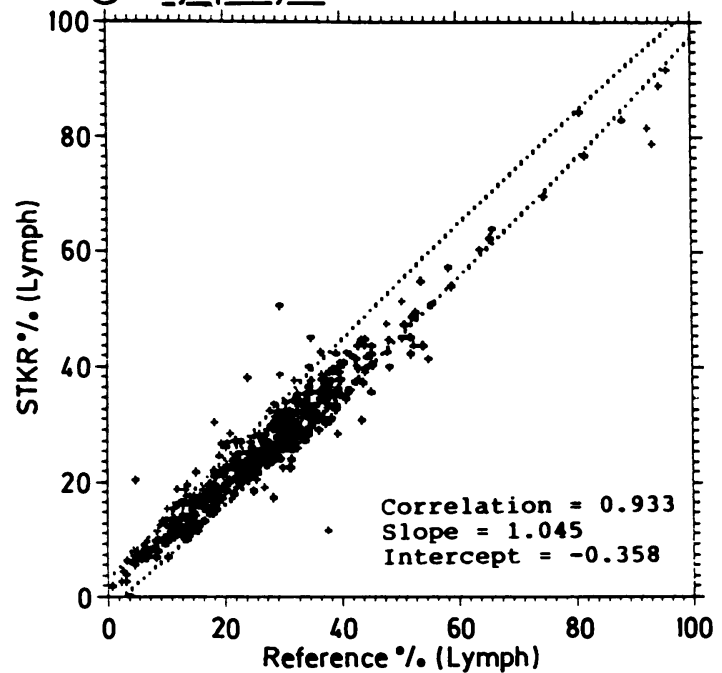

An analysis of workload was performed on randomly selected days before and after implementation of interpretive reporting on the STKR.

\section{Results}

Five hundred and thirty one samples were processed. Twenty four samples did not have results which could be analysed, usually because the white cell count was out of range $\left(>100 \times 10^{9} / 1\right)$. These samples were classified as "positive" on the STKR (see below) but

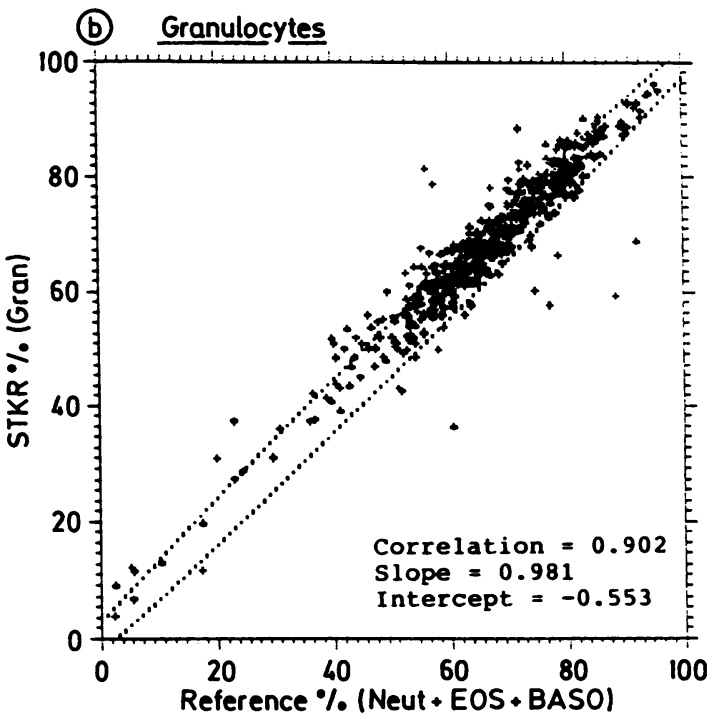

(d) Monocytes

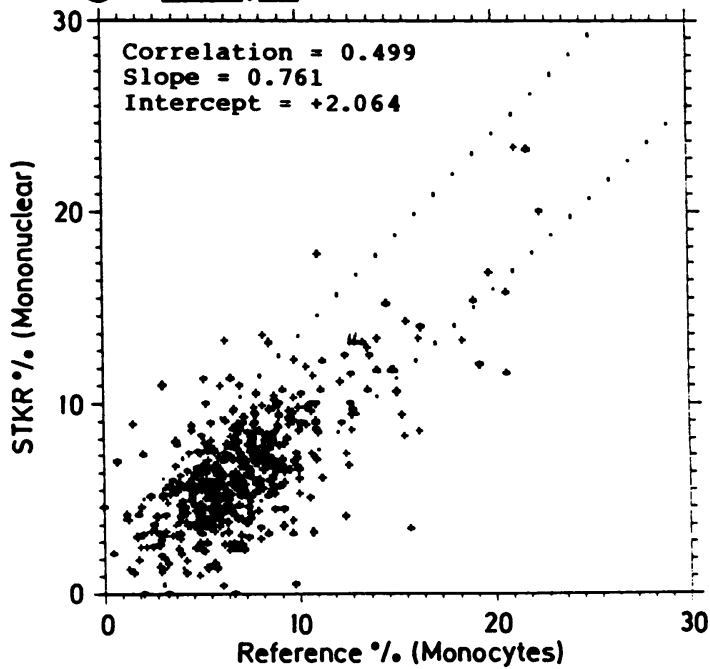

Fig 2 Correlation scatterplots for neutrophils (a), granulocytes (b), lymphocytes (c) and monocytes (d). Each graph also displays the $99 \%$ confidence limits for a 200 cell differential count. 
were not analysed in the part of the study correlating manual and STKR differentials. The range of samples analysed is shown in table 1.

The scatterplots and linear regression statistics for the comparison between the various cell types are shown in figs $2 a-d$. Fig $2 a$ shows the comparison between the mean manual neutrophil percentage and the total granulocyte percentage provided by the STKR. Fig $2 b$ shows the comparison between the mean of the manual total granulocyte percentages (neut + eos + baso) and the total granulocyte percentage provided by the STKR. Fig $2 c$ shows the comparison between the mean manual lymphocyte and the STKR lymphocyte percentage, while fig $2 \mathrm{~d}$ shows a similar comparison for monocytes. Included on the scatterplots are the $99 \%$ confidence limits for a 200 cell differential count-that is, if the count was performed 100 times, counting 200 cells each time, then the results obtained would lie between the confidence limits shown 99 times out of 100 .

Table 2 lists those samples in which the mean manual eosinophil count was $0.7 \times 10^{9} / 1$ or above, with the values for both methods in percentage and absolute numbers shown.

Table 3 lists those samples in which the mean manual basophil count was $0.2 \times 10^{9} / 1$ or greater, with the values for both methods in percentage and absolute numbers shown.

\section{DETECTION OF ABNORMAL CELLS}

Table 4 compares the detection of abnormal cells by manual differential count with the detection by positive flagging on the STKR counter. Of samples processed, $63.6 \%$ were found to be normal by both methods, and $12.4 \%$ were abnormal by both methods. Disagreement between manual and STKR results was found in $24.2 \%$ of samples. False positive STKR results were found in $6.7 \%$ of samples and false negative STKR results in $17.5 \%$ of samples. Seventeen of these 93 false negative samples had immature cell counts of greater than $0.5 \%$. These are listed in table 5 , together with some of the variables routinely provided by the STKR. Abnormal STKR values are boxed. Four of these 17 samples $(4.3 \%$ of all false negative results) had no abnormal features on the blood count that would prompt further examination of the blood film. Table 6 lists the user-defined flagging limits set by this laboratory for out of range results.

Ten of the 507 samples had an NRBC count above 1 per 200 white cells. Five of these samples had a positive STKR flag. In four of these five cases this flag was "suspect eos imm grans" and in one case "suspect NRBC".

EFFECT ON LABORATORY WORKLOAD

Before the use of interpretive reporting on the Coulter
STKR about $40 \%$ of all blood samples required examination of the film. All samples had a blood film made and stained, and this was examined when abnormal or incomplete results were obtained on the blood count. Use of the STKR with interpretive reporting allowed the number of films requiring manual examination to be reduced to about $20 \%$. The making of films is now restricted to those samples that

Table 1 Range of samples tested in this study showing absolute and percentage results of manual 800 cell counts

\begin{tabular}{lll}
\hline & \multicolumn{2}{l}{ Range of samples tested } \\
\cline { 2 - 3 } & $\%$ & $\times 10^{9} / l$ \\
\hline Neutrophils & $0.5-95.8$ & $0.0-55.9$ \\
Lymphocytes & $0 \cdot 8-95.9$ & $0 \cdot 1-81.4$ \\
Monocytes & $0.0-22.4$ & $0.0-4.2$ \\
Eosinophils & $0.0-25.4$ & $0.0-6.0$ \\
Basophils & $0.0-7.6$ & $0.0-4.2$ \\
\hline
\end{tabular}

Table 2 Results from samples with a manual eosinophil count of $0.7 \times 10^{9} / l$ or over

\begin{tabular}{llll}
\hline Flag & $\begin{array}{l}\text { White } \\
\text { cells }\end{array}$ & $\%$ & $\times 10^{9} / l$ \\
\hline+ & 23.7 & 25.38 & 6.0 \\
+ & 76.8 & 6.2 & 4.7 \\
+ & 26.2 & 8.1 & 2.1 \\
+ & 19.6 & 9.4 & 1.8 \\
+ & 7.6 & 12.5 & 0.9 \\
+ & 15.1 & 6.2 & 0.9 \\
- & 7.2 & 10.4 & 0.7 \\
\hline
\end{tabular}

Table 3 Results from samples with a manual basophil count of $0.2 \times 10^{9} / \mathrm{l}$ or over

\begin{tabular}{llll}
\hline Flag & $\begin{array}{l}\text { White } \\
\text { cells }\end{array}$ & $\%$ & $\times 10^{9} / l$ \\
\hline+ & 76.8 & 5.5 & 4.2 \\
+ & 26.2 & 7.6 & 2.0 \\
+ & 15.1 & 6.6 & 1.0 \\
+ & 23.9 & 1.25 & 0.3 \\
+ & 21.2 & 1.4 & 0.3 \\
+ & 14.7 & 2.0 & 0.3 \\
+ & 7.9 & 2.5 & 0.2 \\
+ & 31.3 & 0.6 & 0.2 \\
+ & 9.4 & 2.1 & 0.2 \\
+ & 11.4 & 1.7 & 0.2 \\
- & 6.3 & 3.2 & 0.2 \\
- & 11.8 & 1.7 & 0.2 \\
+ & 33.2 & 0.6 & 0.2 \\
- & 11.4 & 1.7 & 0.2 \\
\hline
\end{tabular}

Table 4 Comparison of normal and abnormal results obtained on 531 samples using Coulter STKR and reference manual method

\begin{tabular}{lll}
\hline & \multicolumn{2}{l}{ Coulter STKR } \\
\cline { 2 - 3 } Reference manual & Positive & Negative \\
\hline Positive & $66(12 \cdot 4 \%)$ & $93(17 \cdot 5 \%)$ \\
Negative & $37(6.7 \%)$ & $335(63 \cdot 6 \%)$ \\
\hline
\end{tabular}


Table 5 Selected STKR blood count results from false negative samples with manual primitive cell count of $>0.5 \%$

\begin{tabular}{|c|c|c|c|c|}
\hline $\begin{array}{l}\text { Percentage } \\
\text { primitive results }\end{array}$ & Haemoglobin & White cells & Platelets & Other \\
\hline $6 \cdot 16$ & $8 \cdot 2$ & $11 \cdot 1$ & 421 & \begin{tabular}{|l|} 
MCV 80.5 \\
\end{tabular} \\
\hline $4 \cdot 49$ & $9 \cdot 4$ & $11 \cdot 8$ & 361 & \begin{tabular}{|l|} 
RDW 18.3 \\
\end{tabular} \\
\hline 3.05 & $12 \cdot 9$ & $13 \cdot 1$ & 188 & LYMPHS $60 \%$ \\
\hline $2 \cdot 17$ & $13 \cdot 2$ & $9 \cdot 2$ & 416 & \\
\hline 1.81 & $9 \cdot 6$ & $10 \cdot 7$ & 359 & \begin{tabular}{|l|} 
RDW 18.4 \\
\end{tabular} \\
\hline 1.8 & $8 \cdot 3$ & $5 \cdot 0$ & 146 & MCV 105 \\
\hline 1.68 & $9 \cdot 2$ & 11.9 & 400 & \begin{tabular}{|l|} 
MCV 99.2 \\
\end{tabular} \\
\hline 1.5 & $11 \cdot 1$ & $8 \cdot 4$ & 489 & \begin{tabular}{|l|} 
MCV 83 \\
\end{tabular} \\
\hline $1 \cdot 01$ & $10 \cdot 8$ & $11 \cdot 5$ & 305 & \\
\hline 1.0 & $10 \cdot 6$ & $7 \cdot 7$ & 290 & \begin{tabular}{|l|} 
MCV 102 \\
\end{tabular} \\
\hline $1 \cdot 0$ & $11 \cdot 9$ & $9 \cdot 4$ & 243 & RDW 15.7 \\
\hline 0.88 & $11 \cdot 9$ & 9.9 & 123 & \begin{tabular}{|l|} 
RDW 19.4 \\
\end{tabular} \\
\hline 0.84 & $14 \cdot 3$ & $6 \cdot 1$ & 38 & \\
\hline 0.82 & $13 \cdot 1$ & $8 \cdot 8$ & 46 & \\
\hline 0.8 & $11 \cdot 2$ & $12 \cdot 4$ & 309 & \\
\hline 0.67 & $13 \cdot 5$ & $7 \cdot 3$ & 504 & \\
\hline 0.55 & $11 \cdot 7$ & $10 \cdot 3$ & 443 & \\
\hline
\end{tabular}

Table 6 User-defined laboratory action limits for this laboratory

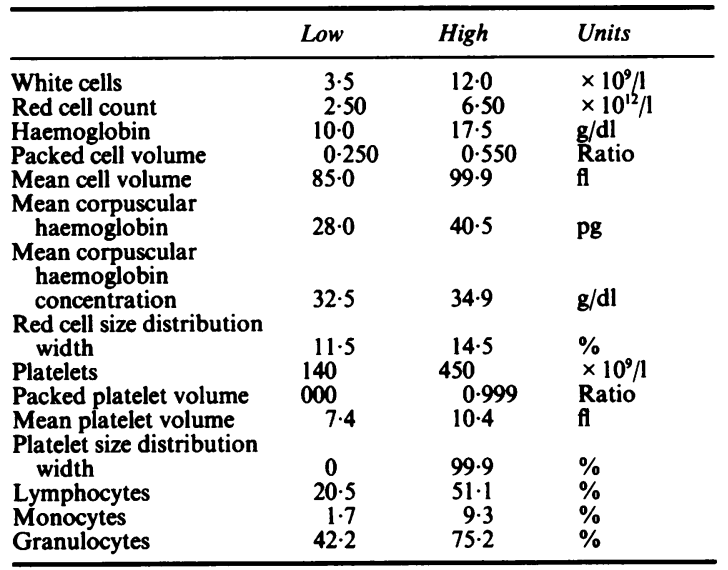

actually require examination. The effect on laboratory workload is shown in table 7.

\section{Discussion}

The scatterplots and linear regression values for the three cell types are satisfactory for neutrophils and lymphocytes. As might be expected, an improved correlation is found when total manual granulocytes
Table 7 Effect on laboratory workload before and after use of interpretive reporting on Coulter STKR

\begin{tabular}{llll}
\hline & $A$ & $B$ & $C$ \\
\hline Before implementation & $23 \%$ & $31 \%$ & $46 \%$ \\
After implementation & $26 \%$ & $52 \%$ & $22 \%$ \\
\hline
\end{tabular}

Column A shows the percentage of counts found to be within normal limits and released to the clinician immediately. Column B shows the percentage of counts with results outside normal action limits (or flagged) but consistent with the clinical details provided and therefore released to the clinician. Column $\mathrm{C}$ shows the percentage of counts where the results were not consistent with the clinical details or contained incomplete data and film examination was performed: this group included about 5\% haematological patients who had manual film examination regardless of blood count results.

are compared with STKR granulocytes. The monocyte scatterplot and values show a significantly worse correlation and this may reflect the difficulties of comparing an automated cell volume analysis with visual interpretation and classification of the large mononuclear cells.

The efficiency of detection for raised eosinophil counts $\left(>0.7 \times 10^{9} / 1\right)$ is shown in table 2 where six of the seven results were flagged as positive. The efficiency of the detection for raised basophils counts $\left(<0.2 \times 10^{9} / 1\right)$ is shown in table 3 where nine of the 14 results were flagged as positive.

Table 5 lists 17 results where the immature cell count is greater than $0.5 \%$, with two of the samples having a 
count above $4 \%$. Critical examination of other variables provided by the STKR, and use of this laboratory's user-defined flagging limits shows that 13 of these results had values that would have prompted this laboratory to undertake an examination of the film. The presence of small numbers of immature cells would not necessarily have been detected on this examination but it is thought that no abnormality of clinical importance is likely to have been overlooked.

In this study 200 cell manual differential counts were performed by experienced observers. In most laboratories a standard manual differential count would require the counting of only 100 cells. Rumkle has drawn attention to the possible errors inherent in counting such relatively small numbers of cells. ${ }^{5}$ Table 4 was constructed assuming that $0.5 \%$ abnormal cells was a reasonable threshold for detection of an abnormality using routine laboratory techniques.

A further safeguard against overlooking clinically important abnormalities is provided by consideration of the clinical details on the request form-for example, counts from patients with known haematological disease would receive a manual differential.
In this laboratory use of the Coulter STKR permitted a $24 \%$ reduction in the number of blood films requiring manual film examination.

\section{References}

1 National Committee for Clinical Laboratory Standards. Leucocyte differential counting. Tentative Standard H20-T 1984;44:11.

2 Hughes-Jones NC, England JM, Norley I, Young JMS. Differential leucocyte counts by volume distribution analysis. $\mathrm{Br} J$ Haematol 1974;28:148.

3 Allen JK, Batjer JD. Evaluation of an automated method for leucocyte differential counts based on electronic volume analysis. Arch Pathol Lab Med 1985;109:534-9.

4 Griswald DJ, Champagne VD. Evaluation of the Coulter S-Plus IV three-part differential in an acute care hospital. Am J Clin Pathol 1985;84:49-57.

5 Rumke CL, Bezemer PD, Kuik DJ. 1975 Normal values and least significant differences for differential leucocyte counts. J Chron Dis 1975;28:661-8.

Requests for reprints to: Dr K G Patterson, Senior Lecturer in Haematology, The Middlesex Hospital, Mortimer Street, London W1N 8AA, England. 